\title{
First Results of POLAR: A dedicated Gamma-Ray Burst Polarimeter
}

\author{
Merlin Kole on behalf of the POLAR collaboration* ${ }^{* \dagger}$ \\ DPNC, University of Geneva \\ E-mail: merlin.kole@unige.ch
}

\begin{abstract}
This year marks the 50th anniversary of the first detection of a Gamma-Ray Burst. Despite intensive research on these transient events many questions remain regarding their progenitors and the processes responsible for their high energy emission. As the existing models for the prompt emission have largely differing predictions on the polarization parameters, polarimetric measurements are key to the study of Gamma-Ray Bursts. POLAR is a dedicated gamma-ray polarimeter designed to produce the first catalog of polarization measurements of the prompt emission of Gamma-Ray Bursts. The instrument was launched successfully from the Jiquan Satellite Launching Centre in Inner-Mongolia, China, on the 15th of September 2016. POLAR measures the polarization by exploiting the dependency of the azimuthal Compton scattering angle of incoming gamma-rays on the polarization vector of the photons. The azimuthal Compton scattering angle is measured using a finely segmented scintillator array consisting of 1600 plastic bars with a surface area of 6 by $6 \mathrm{~mm}$ and a length of $176 \mathrm{~mm}$. They are read out in groups of 64 by 25 flat-panel multi-anode photomultipliers. Due to its large granularity POLAR can measure the photon interaction locations, and therefore the scattering angles, with a high precision resulting in a relatively high modulation factor. The instrument furthermore has a relatively large effective area and a field of view of $1 / 2$ the sky, as a result POLAR is one of the most sensitive detectors currently in orbit in its energy range. A total of 55 Gamma-Ray Bursts as well as the Crab pulsar have been detected by POLAR during the first 6 months and searches for electromagnetic counter parts of gravitational waves as well as high energy neutrino counter parts have been performed during this period. An overview of the POLAR detector will be presented along with the first result of the in-flight performance. Finally the measured Gamma-Ray Bursts will be discussed together with prospects for polarization measurements of these events and the future prospects of the experiment.
\end{abstract}

35th International Cosmic Ray Conference - ICRC2017

10-20 July, 2017

Bexco, Busan, Korea

*Speaker.

†As presented: on isdc.unige.ch/polar/ 


\section{Introduction}

To date few polarization measurements of the high energy emission from astrophysical sources exist. While the flux, spectral and timing measurements of $\gamma$-ray emission has been studied extensively for a large variety of astrophysical sources, the two remaining parameters of the emission, the polarization degree and angle, remain largely unmeasured. The polarization properties of the $\gamma$-ray emission is largely dependent on the emission processes involved and is therefore a probe of the emission environments. Polarimetry therefore holds a large potential to study high energy emission environments such as neutron starts, X-ray binary systems, active galactic nuclei, solar flares and Gamma Ray Bursts (GRBs).

Due to the large scientific potential in the field of polarimetry a number of new missions has been proposed dedicated to measure the polarization parameters. Currently several new dedicated polarimeters in the $\mathrm{X} / \gamma$-ray energy range are under construction, some examples are $\mathrm{X}$-Calibur [1] and eXTP [2] while several smaller scale missions, such as GAP [3][4], COSI [5] and PoGOLite [6] have performed polarization measurements in recent years. Furthermore a number of instruments without a dedicated polarimeter have managed to perform polarization measurements of astrophysical sources in the X/ $\gamma$ ray energy range. Examples of this are the IBIS and SPI instruments on the Integral mission which for example both published polarimetric measurements of the Crab pulsar [7] [8]. Such measurements have however in generally been non-constraining.

The POLAR instrument [9] is a new dedicated polarimeter which was launched as part of the Chinese Tiangong 2 (TG-2) Space Lab in September 2016. The expected lifetime of POLAR will be equal to that of TG-2 which is foreseen to operate for at least 2 years. The instrument makes use of a highly segmented scintillator array optimized for performing polarization measurements in the $50-500 \mathrm{keV}$ energy range, while its wide field of view optimizes it for transient sources. In this paper the scientific goals of POLAR are briefly discussed first, followed by a detailed description of the polarimeter. This is followed by a description of the in-flight performance after launch together with the highlights of the measurements performed so far. Finally the future prospects for polarization measurements and the results expected from the remainder of the mission are discussed.

\section{GRBs}

GRBs consist of strong short flashes of $\mathrm{X} / \gamma$-rays, called the prompt-phase, followed by a longer lasting afterglow emitted at lower energies. GRBs can be divided into two different types differing in the length of the prompt-emission. Short burst are thought to originate from the merger of two compact objects such as neutron starts, whereas the longer bursts are thought to originate from the collapse of a massive star. In most of the existing emission models the high energy emission from both long and short GRBs originates from two jets, as a result bursts can only be measured when the jet is within the line of sight of the observer.

Polarization measurements are capable of probing several aspects of astrophysical jets and can therefore be used to answer some of the many questions concerning the gamma-ray emission mechanisms and environments of GRBs. The polarization degree of the $\gamma$-rays largely depends on the emission mechanism of the photons. Models containing emission through synchrotron radia- 
tion in a strong magnetic field predict large polarization degrees as high as 50\% [10]. The exact polarization degree will depend on the viewing angle of the observer with respect to the jet. In the case of highly ordered magnetic fields the average polarization degree will be $40 \%$ within a sample of GRBs. In other models where the emission is mostly a result of inverse-Compton scattering the polarization degree is predicted to only be high in case of a large angle between the observer and the jet. These models predict a strong negative correlation between the observing angle the and polarization degree [11].

In case of several models the polarization angle can furthermore be used to probe the internal structure of the emission jet. Different models exist regarding the structure, some predict an axisymmetric structure, whereas others predict a jet consisting of several mini-jets. An axi-symmetric model makes the strong prediction that the polarization angle of the emission can only change during the observation by $90^{\circ}$, or it can not change at all [12]. For models with mini-jets the observer measures the polarization angle resulting from the magnetic field in the strongest minijet in the field of view. As the jet evolves a different mini-jet can be observed and therefore the measured polarization angle is allowed to vary in any possible way. In recent years the GAP instrument has measured one long GRB during which there is strong evidence that the angle varies during the burst by $90^{\circ}$ [3]. However, the error on the variation of the angle are rather large. Furthermore to confirm an axi-symmetric model this would have to be observed for a large sample of bursts, a catalog of measurements is therefore required. The POLAR detector was developed to provide such a catalog by accurately measuring the polarization degree of several GRBs per year.

\section{POLAR}

POLAR is a space-borne Compton polarimeter with a total of 1600 readout channels. The 1600 channels are divided into 25 independent detector modules, three central FPGAs take care of the overall trigger logic, readout and the communication between the 25 modules. Each detector module consists of 64 scintillator bars connected to a single multi-anode photomultiplier (MAPMT) which in turn is connected to its own front-end electronics which handles both the trigger logic of the module and the communication with the central FPGAs. The scintillator bars, which have a size of $5.8 \times 5.8 \times 176 \mathrm{~mm}^{3}$, are wrapped in Vikuiti to improve the light yield and to reduce the optical cross-talk between channels. In order to protect for vibrations during launch the scintillators are covered at the top by thin rubber dampers. An elastic optical coupler between the plastic scintillators and the MAPMT provides additional protection. A single detector module is shown in figure 1. The full flight model is shown in figure 2. Photons in the $50-500 \mathrm{keV}$ energy range entering the instrument have a high probability to undergo Compton scattering in one of the scintillator bars. Subsequently the photon can interact in a second scintillator bar either through Compton scattering or through photoelectric absorption. This is schematically shown in figure 3 . The azimuthal Compton scattering angle of the photon can be reconstructed by using the position of the two scintillator bars in which an energy deposition took place. The azimuthal Compton scattering angle holds information on the initial polarization angle of the photon as described in the Klein-Nishina equation [13]: 


$$
\frac{\mathrm{d} \sigma}{\mathrm{d} \Omega}=\frac{\mathrm{r}_{0}^{2}}{2} \frac{\mathrm{E}^{\prime 2}}{\mathrm{E}^{2}}\left(\frac{\mathrm{E}^{\prime}}{\mathrm{E}}+\frac{\mathrm{E}}{\mathrm{E}^{\prime}}-2 \sin ^{2} \theta \cos ^{2} \phi\right)
$$

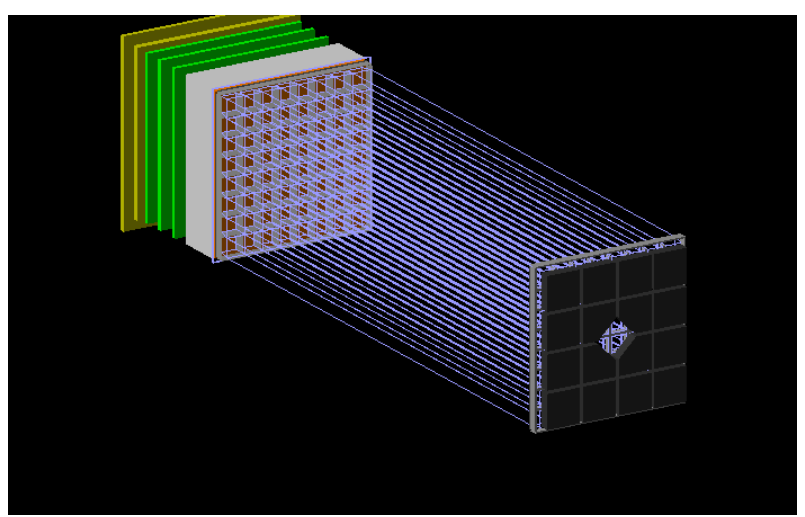

Figure 1: Schematic representation of a single module of POLAR. The scintillator bars, shown in blue, together with the MAPMT (grey), the front-end electronics (green) and the rubber dampers on top (black).

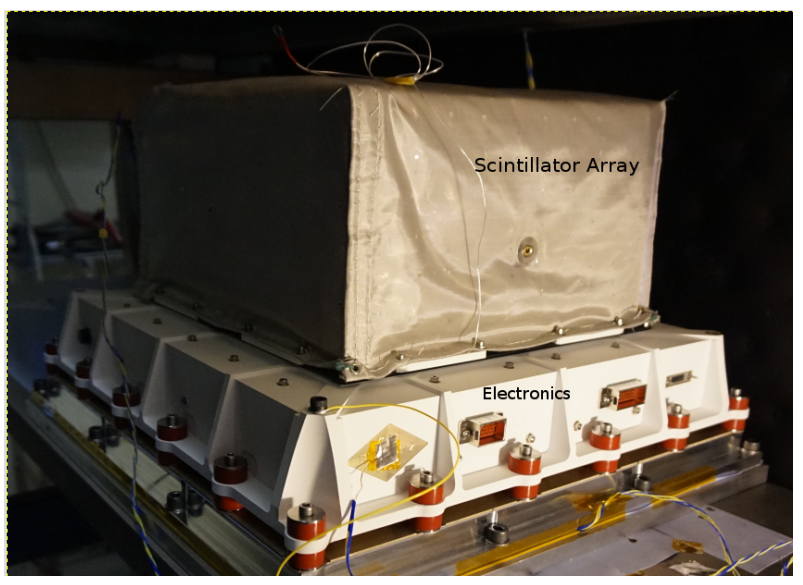

Figure 2: The POLAR flight model during thermal vacuum tests. The electronics is contained in the white painted aluminum structure while the scintillator array is placed in a carbon structure covered with a multilayer insulator.

Here $r_{0}^{2}$ is the classical electron radius, $E$ is the initial photon energy, $E^{\prime}$ the final photon energy, $\theta$ the polar scattering angle and $\phi$ the azimuthal Compton scattering angle with respect to the polarization vector as visualized in figure 3 . The $\cos ^{2} \phi$ term causes the photons to preferentially scatter perpendicular to their initial polarization vector. For a polarized photon flux Compton scattering in POLAR this results in a non-uniform distribution of the azimuthal scattering angles. The distribution of azimuthal scattering angles can be described by a function of the form:

$$
f(\phi)=A(1+\mu \cos (2(\eta-\phi)+\pi)
$$

Here $\mathrm{A}$ is the mean of the harmonic function, $\phi$ represents the angle between the polarization vector of the incoming photon and the scattering angle, $\eta$ is the measured scattering angle, and $\mu$ 
is referred to as the modulation factor. The polarization degree can be retrieved from $\mu$ by dividing it by $\mu_{100}$ which is the instrument's response to a $100 \%$ polarized beam. The value of $\mu_{100}$ is both instrument and energy dependent. As a result this value needs to be acquired using a combination of calibration measurements and simulations. The accuracy with which a polarization measurement can be performed can be expressed using Minimal Detectable Polarization (MDP). This parameter represents the minimum degree of polarization required in order to be able to confirm through measurement that the observed signal is polarized within a set confidence level. In this paper all the MDPs stated assume a confidence level of $3 \sigma$.

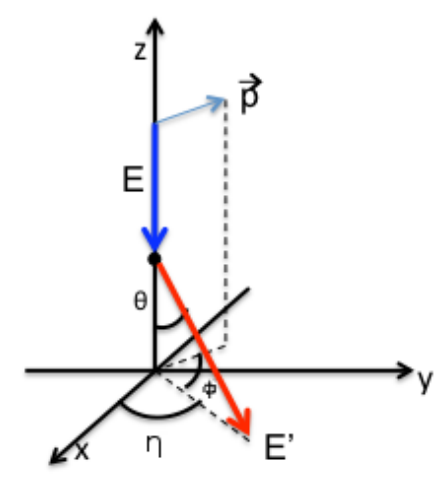

Figure 3: Schematic representation of the Compton scattering process. The incoming photon (blue; energy E, polarization $\vec{p}$ ) scatters off an electron with a polar angle $\theta$ from its original trajectory. $\phi$ is the angle between the polarization vector of the incoming photon and the azimuthal scattering angle $\eta$.

The choice of a full plastic scintillator array for POLAR results in a relatively high cross section for Compton scattering for photons in the lower part of the instruments energy range while ensuring a relatively large effective area. The uniform detection surface furthermore results in a wide field of view of half the sky. Although the value of $\mu_{100}$ drops off towards large off-axis angles polarization measurements can be performed accurately for GRBs within approximately $1 / 3$ of the sky [14]. In order to acquire the dependency of $\mu_{100}$ on energy and incoming photon angle and to fully understand the instrumental effects on the modulation curve the instrument underwent dedicated calibration tests prior to launch [15]. Using the results of these calibration tests a detailed Monte Carlo based simulation package was produced which accurately describes the instruments response to both polarized and unpolarized beams with different energies and for different incoming angles [16].

\section{In-flight Performance}

POLAR was launched on the 15th of September 2016 from the Jiquan Satellite Launching Centre in Inner-Mongolia China as part of the Tiangong 2 (TG-2) space laboratory. The instrument is placed on TG-2 such that it always points to zenith, therefore POLAR continuously observes half of the sky. The instrument was successfully switched on on the 23rd of September 2016 after which it has been almost continuously taking data. All 1600 channels were found to operate normally 
while the noise levels observed in-orbit are largely equal to those measured during pre-launch tests. Background levels and their dependencies on the position of TG-2 with respect to Earth were furthermore found to be compatible with previous predictions presented in [14]. Despite not being optimized for point source observations, the Crab pulsar was measured using the data from the first six months of flight [17]. The pulsar profile, as well as the pulsar profile as a function of the observation time can be observed in figure 4. The observation of the Crab illustrates the timing of POLAR to be accurate withing $1 \mathrm{~ms}$. This was confirmed using measurements with the InterPlanetary Network where the arrival time of a GRB at the POLAR detector was found to be in agreement with the time expected using measurements of the GRB by other instruments. Despite several periods during which the instrument had to be switched off as requested by the General Establishment of Space Science (GESSA) POLAR measured a total of at least 55 GRBs during the first 6 months of observations [18]. A total of 49 of these GRBs were reported to the community through the Gamma-ray Coordinates Network (GCN) and all were confirmed through observations of other instruments. Currently the data is being analyzed in order to search for GRBs only observed by POLAR.

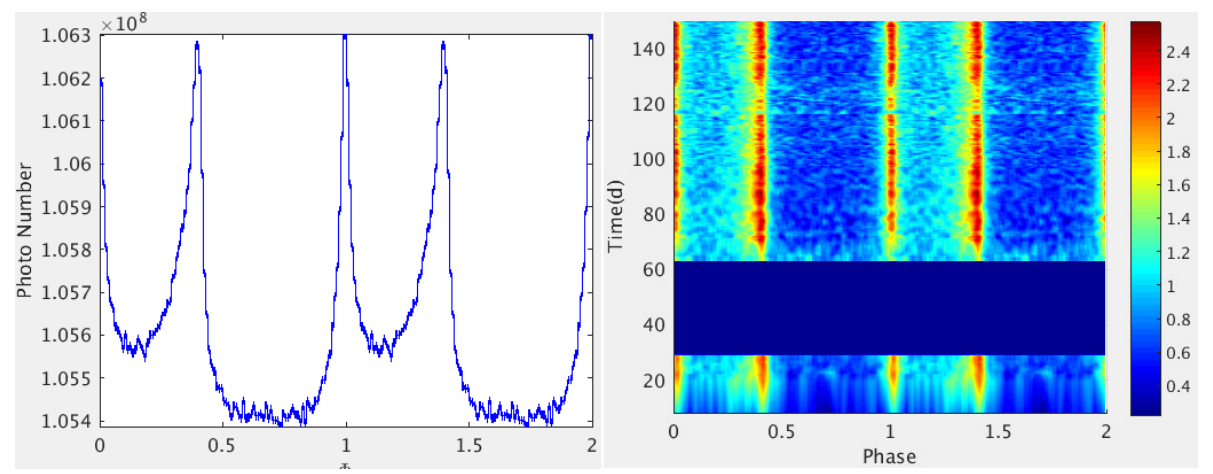

Figure 4: The pulsar profile of the Crab as measured by POLAR on the left together with the pulsar profile as a function of time (in days since switching on the instrument) on the right. The area without data visible in the right hand figure coincides with the period during which POLAR was switched off due to the presence of taikonauts on board TG-2.

The nominal data product of POLAR consists only of events which can be used for polarization studies. Such events are required to have energy deposition in at least two bars making it likely a result a photon interacting at least twice within the active volume. During the early phase of 2017 the data size on TG-2 reserved for POLAR was increased allowing for the additional storage of events consisting of only one triggered bar. As a result the sensitivity of POLAR to detect $\gamma$-ray emission was greatly increased allowing this data to be used to search for electromagnetic counter parts of gravitional wave (GW) events, high energy neutrinos and fast radio bursts. During this period which started in January 2016 ended in April 2017 the effective area of POLAR in the $20-500 \mathrm{keV}$ energy range was found through simulations to be approximately $300 \mathrm{~cm}^{2}$, making it one of the most sensitive detectors in this energy range.

\section{GRBs Measurements and Future Prospects}

To date POLAR has a total of 55 confirmed GRB detections. Examples of light curves of 2 
GRBs measured by POLAR, 170202B and 170127C, are shown in figures 5 and 6 respectively. It should be noted here that the light curve of $170127 \mathrm{C}$ contains also events with only 1 triggering bar as discussed in the previous section, while the light curve of 170202B contains only events which can be used in polarization studies. The light curves of all confirmed GRBs observed by POLAR can be found on http://www.isdc.unige.ch/polar/lc/. Preliminary studies of the statistics acquired for all GRBs shows that approximately 10 of the observed GRBs are good candidates for polarization studies, meaning an MDP lower than 30\%. Approximately 3 of the measured GRBs have an MDP lower than 15\% allowing for measurements with an unprecedented precision of their polarization properties. Detailed on-ground calibration studies of the instrument, required for the polarization studies were finalized during early 2017 while in-orbit calibration is currently in the final stages [16] [19] [20]. The finalization of these studies, which will result in a proper understanding of the systematic errors of the analysis will be finalized in the coming months and will be followed by analysis of the polarization of the first sample of GRBs. Extrapolating from the number of GRBs observed during the first months of operation the number of GRBs observed by POLAR during the expected lifetime will be around 300 while significant polarization measurements can be expected for approximately 50 GRBs. Furthermore searches for EM counter parts of for example GWs in the existing data as well as in the new data are ongoing.

POLAR-GRB 170202B (125 ms bins)

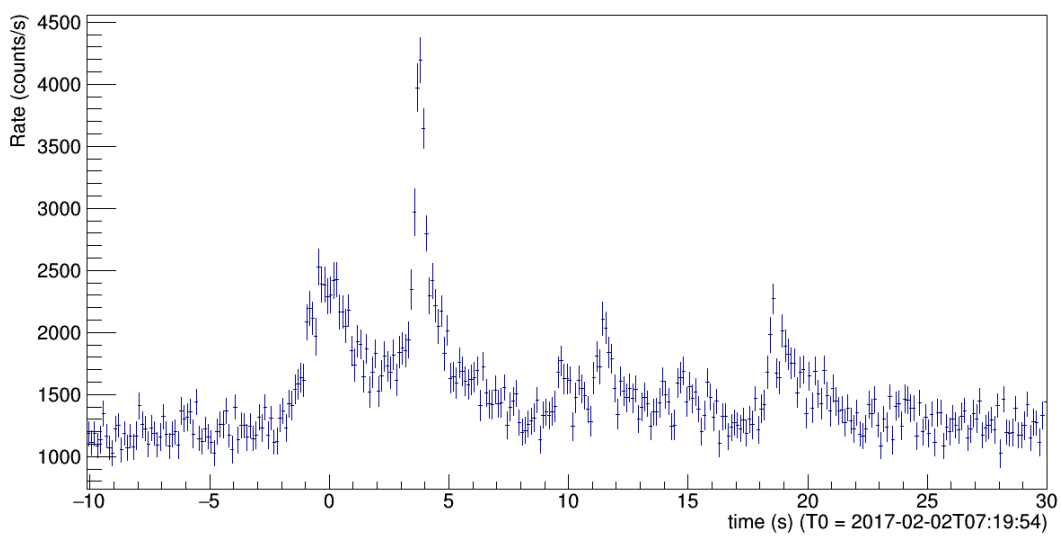

Figure 5: The light curve of GRB 170202B as measured by POLAR

\section{Conclusions}

POLAR is a dedicated polarimeter designed to measure the polarization of the prompt emission of GRBs in the $50-500 \mathrm{keV}$ energy range. The detector was launched successfully as part of TG-2 on the 15th of September 2016. After switching on the instrument on the 23rd of September the performance was found to be in agreement with previous predictions. A total of 55 GRBs have been confirmed to be measured to date. The sample of measured GRBs contains approximately 10 GRBs for which a measurement with an MDP below 30\% can be achieved, while for about 3 of these candidates an MDP below 15\% can be achievable. The instrument was furthermore shown to be capable of being one of the most sensitive detectors in its energy range allowing it search for electromagnetic counter parts of for example GWs. 


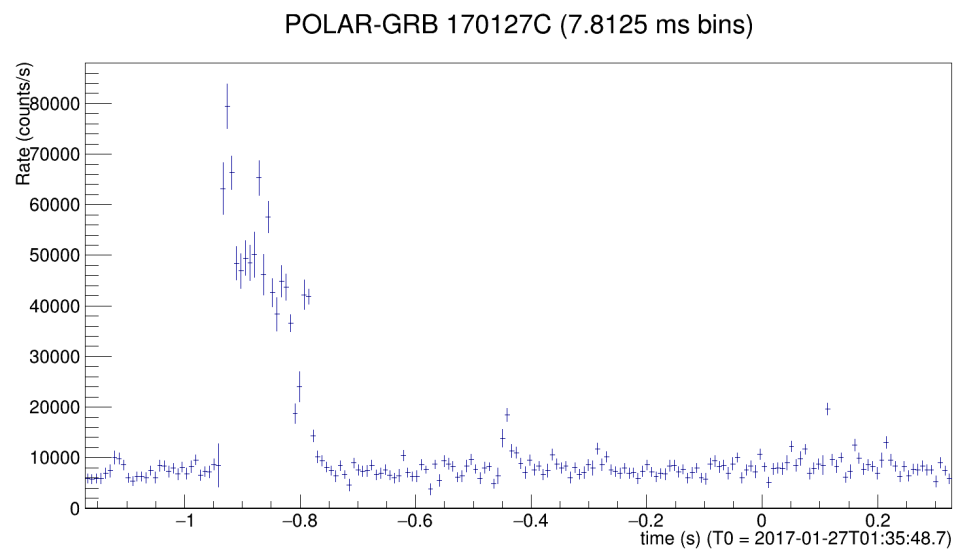

Figure 6: The light curve of GRB 170127C as measured by POLAR. It should be noted here that this light curve also contains events with only 1 triggering bar which cannot be used for polarization studies.

\section{References}

[1] Q. Guo et al., ApJ. 41 (2013) 63

[2] S.N. Zhang et al., SPIE proc. Vol 9905 arXiv:1607.08823

[3] D. Yonetoku et al., ApJ. 743 (2011) 30

[4] D. Yonetoku et al., ApJ. 758 (2012) 1

[5] C.A. Kierans et al., Proc. INTEGRAL Conf. arXiV:1701.05558v1

[6] M. Chauvin et al., MNRAS 456 (2016) 84-88

[7] M. Chauvin et al., ApJ. 769 (2013) 137

[8] M. Forot et al., ApJ. 688 (2008) 29

[9] N. Produit et al., Nucl. Instr. and Meth. A 550 (2005) 616.

[10] M. Luytikov et al., ApJ. 597 (2003) 998

[11] S. Dado et al., Proc. Vulcano Conf. arXiv:astro-ph/0701294v2

[12] C. Lundman et al., MNRAS 428 (2013) 2430

[13] O. Klein, Y. Nishina, Z. Phys. 52 (1929) 853

[14] E. Suarez, Doctoral Thesis, Univ. of Geneva 2010, at http://archive-ouverte.unige.ch/unige:6889

[15] M. Kole et al., ICRC Conf. Proc. 2015

[16] M. Kole et al., submitted to Nucl. Instr. and Meth. A

[17] Li H. C., Ge, M. Y., et al, ICRC Conf. Proc. 2017

[18] Xiong S.L. et al., ICRC Conf. Proc.2017

[19] Wang Y.W. et al., ICRC Conf. Proc. 2017

[20] Xiao H.L et al., ICRC Conf. Proc. 2017 